\title{
Montée de l'immunité profonde : vers l'équilibre endémique du Sars-CoV-2
}

\author{
Samuel Sender $(P h D)^{1}$
}

\begin{abstract}
Pour les virus respiratoires, c'est l'immunité profonde, c'est-à-dire la réduction de la sévérité des infections virales, qui mesure le chemin parcouru vers la phase endémique.

La sévérité pourrait se mesurer de manière continue comme ratio entre les nouvelles formes sévères (hospitalisations, décès) et les nouvelles infections, si une mesure fiable et continue des nouvelles infections existait, ce qui n'est pas le cas.

Nous nous basons sur la théorie immunologique pour construire un indicateur de progression de l'immunité profonde dans la population. Cet indicateur, basé sur la déformation de la structure d'âge des hospitalisations Covid, est construit en temps réel et tient compte de l'impact du passage du temps, des variants et de la vaccination.

Nous montrons que plus des deux tiers du chemin qui caractérise le passage à l'endémie du Sars-CoV2 a été parcouru, avec une progression des deux-tiers de l'immunité profonde chez les 30-60 ans. La mesure de la progression de l'immunité profonde dans les autres classes d'âge est moins fiable, mais aussi moins pertinente car :

- Les moins de 30 ans avaient virtuellement tous acquis avant la première vague une immunité croisée profonde par exposition aux coronavirus endémiques. Leur immunité ne peut pas progresser de manière marquée.

- L'immunité des plus de 60 ans n'étant dans pas robuste, la mesure de sa progression est hasardeuse ; l'immunité dans cette classe d'âge est appelée à être renforcée par la vaccination.
\end{abstract}

Notre indicateur et la théorie immunologique sous-jacente permettent d'expliquer les développements récents des caractéristiques de l'épidémie : circulation et sévérité saisonnière, une plus forte circulation chez les plus jeunes, et statistiques globales de mortalités qui sont redescendues vers les moyennes saisonnières de long-terme.

\section{Keywords: coronavirus, Sars-CoV-2, Covid, Covid19, estimation, endémie, immunologie, immunité profonde JEL codes: $C 13, C 18, C 52,11,118$}

\footnotetext{
${ }^{1}$ Professeur associé, économétrie et finance ; consultant. https://www.linkedin.com/in/samuel-sender-phd/ Les graphiques de cette étude sont mis à jour quotidiennement ici : https://samjs.sjinyapps.io/CoViD Je remercie Pierre Sonigo, qui par nos discussions très riches sur la structure d'âge de la mémoire et réactivité immunitaire a permis de créer cet indicateur.

Pierre Sonigo, Chief Scientific Officer des laboratoires Sebia : https://www.linkedin.com/in/pierre-sonigo/
} 


\section{Table of Contents}

Résumé 3

Le problème de mesure $\quad 3$

Théorie immunologique 3

Méthodologie $\quad 4$

Résultats et interprétation $\quad 4$

Introduction $\quad 5$

I) De l'épidémie à l'endémie : le cas des coronavirus 5

Un passage naturel à l'endémie $\quad 5$

$\begin{array}{ll}\text { Qu'est-ce que l'endémie pour un virus respiratoire ? } & 6\end{array}$

II) Nouvelle stratégie d'estimation de l'immunité collective et indicateur en temps réel 7

Le manque de fiabilité des mesures d'exposition et la nécessité de mesures alternatives $\quad 7$

Préambule : évidence d'une structure d'âge des formes sévères $\quad 8$

$\begin{array}{lr}\text { Estimation pour la France } & 9\end{array}$

$\begin{array}{lr}\text { Cohérence et biais de l'estimateur } & 10\end{array}$

Robustesse 11

III) Analyse de long-terme : limites et données alternatives 12

Limites des données d'hospitalisation $\quad 12$

Séries longues 12

$\begin{array}{ll}\text { Conclusion } & 13\end{array}$

Appendices $\quad 15$

A.2) Saisonnalité des virus aériens et du Sars-CoV2 15

Références 16 


\section{Résumé}

\section{Le problème de mesure}

Les virus rhumo-grippaux ne disparaissent pas mais ils évoluent conjointement avec le système immunitaire de leurs hôtes et acquièrent des propriétés stables dans la phase dite endémique.

Pour les virus aériens, le chemin vers l'endémie est caractérisé par la progression de l'immunité profonde, c'est-à-dire la diminution de la sévérité des infections virales.

La sévérité pourrait se mesurer comme le ratio du nombre de nouveaux cas graves (hospitalisation ou décès) sur le nombre de nouveaux cas (nouvelles infections), se celles-ci étaient mesurées de manière fiable et continue.

Mais les estimations des nouvelles ne sont pas fiables : les tests PCR souffrent d'un biais de participation, et les enquêtes sérologiques ne sont pas fréquemment répétées ; par ailleurs, les deux mesures sont biaisées.

\section{Théorie immunologique}

La théorie immunologique permet de décrire la transition vers l'endémie :

- la phase épidémique se caractérise par une forte sévérité (hospitalisations et décès) potentiellement dans des tranches d'âges assez larges

- la phase endémique se caractérise par l' immunité profonde d'une fraction importante et stable de la population; l'immunité acquise profonde n'empêche pas la circulation du virus.

- le virus quant à lui virus touche plus les voies aériennes supérieures en réaction à l'immunité profonde ; il devient plus contagieux

Cette transition peut être caractérisée par une modification de la structure d'âge des infections graves, comme de la circulation des virus aériens :

- la circulation devient plus intense chez les jeunes enfants (au-delà des modifications immulogiques et virales décrites ci-dessus, dans la phase endémique, le primo-infections finissent par concerner les plus jeunes enfants)

$\circ$ une population adulte qui par son exposition répétée, acquiert une forte immunité profonde (jusqu'à son niveau de long-terme)

- l'immunité acquise fragile, voire défaillante des population âgées est partiellement compensée par l'immunité vaccinale.

Pour résumer, la transition vers l'endémie se caractérise par une pentification progressive de la (partie longue de la) structure d'âge des hospitalisations, avec une population des 30-60 ans globalement moins impactée dans la phase endémique quand dans la phase épidémique, alors que les plus de 60 ans restent fragiles. 


\section{Méthodologie}

Dans certains pays comme la France et les Etats-Unis, la structure par âge des formes sévères est accessible, et les données d'hospitalisation sont mises à jour quotidiennement.

Nous construisons un indicateur instantané de la sévérité relative du virus, qui suit l'évolution de la structure d'âge des hospitalisations. Il s'agit du ratio des 30-60 ans hospitalisés rapporté aux 60 + hospitalisés, normé à 100 a pic de la première vague. Nous prenons compte de l'immunité vaccinale à partir de 2021.

\section{Résultats et interprétation}

Nous montrons une progression de l'immunité profonde dans plus des deux-tiers de la population active ; ceci n'implique non seulement une exposition d'au moins les deux-tiers de cette population, mais aussi l'absence d'échappement majeur des variants (jusqu'à présent) à l'immunité vaccinale.

Les nouveaux variants semblent ralentir la progression de l'immunité profonde, témoignant d'un échappement partiel ; à l'inverse, la vaccination semble avoir bénéficié aux plus âgés.

\section{Limites et données alternatives}

L'endémie ne peut se juger que sur des tendances de long-terme.

Des données d'hospitalisations de long-terme seraient nécessaires. Elles permettraient d'évaluer si la vague épidémique actuelle s'écarte significativement de la saisonnalité typique des virus rhumo-grippaux (auxquels appartiennent les coronavirus endémiques).

Cette analyse doit être conduite sur des données alternative longues, et la seule disponible est la mortalité toutes causes confondues. Les données récentes montrent que la mortalité toute causes confondues est redescendue vers les tendances saisonnières de long terme, pour toutes les groupes d'âge.

Cela peut être interprété comme le résultat conjoint de l'augmentation de l'immunité acquise pour la population en âge de travailler et de l'augmentation de l'immunité vaccinale pour la population âgée.

Les données de mortalité suggèrent donc une transition progressive vers une phase endémique dans laquelle le Sars-CoV-2 aurait un impact similaire à celui d'autres virus aériens. 


\section{Introduction}

Après une année d'épidémie, les politiques et les acteurs économiques doivent évaluer à quel moment le Sars-CoV2 pourra être catégorisé comme virus endémique au même titre que la grippe saisonnière et les quatre coronavirus endémiques. ${ }^{2}$

Nous décrivons d'un point de vue immunologique le passage de la phase épidémique à la phase endémique ; si le passage vers l'endémie est attendu, il est crucial de mesurer son avancement.

Nous estimons que dans la population active susceptible d'être touchée sévèrement par la COVID, les deux tiers ont déjà acquis une immunité profonde, et la sévérité empirique a ainsi diminué des deux tiers.

L'immunisation profonde des moins de 30 ans (par exposition aux coronavirus endémiques cousins) étant déjà presque totale dès le début de l'épidémie, il n'est pas possible de mesurer de manière fiable la progression de l'immunité dans cette tranche d'âge (mais cette mesure importe peu).

La montée de l'immunité diminue les risques d'une flambée exponentielle de la mortalité, et le retour de la mortalité vers les tendances saisonnières de long-terme semble confirmer l'approche de la phase endémique.

\section{I) De l'épidémie à l'endémie : le cas des coronavirus}

Un regard immunologique sur les modèles épidémiologiques

Un passage naturel à l'endémie

Commençons par une vue d'ensemble très large.

Les virus ont des caractères intrinsèques, liés à leur capacité à se transmettre facilement (c'est le cas des virus respiratoires), à leur capacité d'accroche aux récepteurs cellulaires (c'est le cas du Sars-CoV2 avec la protéine Spike sur les récepteurs ACE2), à leur capacité de multiplication dans certains organes ou types cellulaires donnés, puis à leur capacité à endommager ces organes, soit directement (dégradation des cellules), soit indirectement (inflammation, action du système immunitaire).

Toutefois, la pathogénicité d'un virus n'est pas une caractéristique intrinsèque du virus mais de sa relation à l'hôte. C'est le résultat conjoint des caractéristiques intrinsèques du virus et des défenses immunitaires acquises par première exposition au virus (ce que l'on appelle l'immunité acquise homologue), par exposition à des virus proches (immunité acquise croisée), ou par vaccination (immunité vaccinale).

Pour les virus aériens dont le potentiel de circulation est très élevé, on arrive naturellement à une exposition progressive de la majorité de la population, ce qui en retour permet la construction de défenses immunitaires dans la population.

\footnotetext{
${ }^{2}$ Les quatre coronavirus humains (HCoV) responsables d'infections courantes chez l'homme : HCoV-OC43, HCoV-229E, HCoV-NL63 et HCoV-HKUl, voir Segondy (2020) pour une description.
} 
Il est important de préciser qu'en virologie, les vaccins ne font jamais mieux que l'infection elle-même. Il est donc habituellement inutile de vacciner les personnes déjà infectées.

La protection immunitaire est d'autant plus forte que l'infection (ou la vaccination) est récente et que le premier virus infectant est peu différent de celui qui arrive ensuite.

Ainsi, l'infection par les coronavirus endémiques qui survient chez $100 \%$ de la population dans l'enfance autour de l'âge de 3 ans protège très bien contre Sars-CoV2, malgré la forte différence entre le virus immunisant et le virus qui vient en second, pendant la décennie qui suit, c'est-àdire les enfants et les sujets jeunes.

Cette protection devient insuffisante lorsqu'on avance en âge : à la baisse naturelle de l'immunité acquise avec le temps s'ajoute la différence entre le premier et le second virus.

\section{Qu'est-ce que l'endémie pour un virus respiratoire?}

Pour de nombreux virus qui ne touchent pas les voies respiratoires, l'immunisation d'un individu et l'élimination du virus dans le corps rend cet individu non contagieux, et non susceptible d'être réinfecté, ce qui réduit la circulation du virus et permet son éradication.

Le modèle épidémiologique standard SIR décrit l'éradication par immunité collective : au début de l'épidémie, chaque personne infectieuse contamine R0 nouvelles personnes ; quand plus de la fraction 1-1/R0 de la population a été immunisée et qu'une proportion inférieure à 1/R0 est susceptible, chaque personne infectée contamine moins d'une personne.

Pour un virus respiratoire, il n'est pas possible de parler d'immunité de groupe bloquant la transmission comme pour la variole ou la poliomyélite. Il est essentiel de distinguer l'immunité profonde qui protège des formes graves touchant les poumons et l'immunité muqueuse qui bloque la transmission. En effet, l'immunité muqueuse est peu efficace, et transitoire, comparée à l'immunité profonde. Elle n'empêche pas la circulation du virus via l'infection bénigne des voies respiratoires supérieures. Il s'agit de sinusites ou rhinopharyngites couramment appelées rhumes.

Ainsi, la population est depuis longtemps largement immunisée contre les maladies graves provoquées coronavirus endémiques ou la grippe, mais cela n'empêche nullement le virus de circuler via des infections le plus souvent bénignes.

Sur la question de la durée de la protection résultant d'une infection passée, il est essentiel de distinguer les réinfections bénignes survenant sous forme de rhumes et les infections des tissus profonds comme le poumon. La protection contre les rhumes est mauvaise et les réinfections bénignes surviennent malgré une excellente protection contre les infections profondes. Sans effectuer cette distinction, la question de la durée de l'immunité ou de la protection contre la transmission deviennent extrêmement confuses.

L'endémie se caractérise du point de vue statistique comme un état stationnaire, ${ }^{3}$ c'est-à-dire que ses caractéristiques sont stables. D'un point de vue statistique, l'endémie ne peut se

\footnotetext{
${ }^{3}$ Un processus est dit stationnaire si ses propriétés ne dépendent pas du temps ; pour simplifier, dans le cas de virus à priori saisonniers, un processus est stationnaire si ses propriétés « mesurables » telles que nombre de contaminations et de morts attendus pour une année quelconque sont égales à leur moyenne de long-terme. Pour une présentation basique, voir Wikipedia en français ici, et pour une présentation plus technique, en anglais ici.
} 
constater qu'ex-post, quand le passage des années permet de caractériser la moyenne et variance du nombre de morts.

D'un point de vue pratique, la situation est donc endémique quand les virus aériens circulent largement dans la population (immunité faible des muqueuses) avec des effets acceptables (immunité profonde forte pour la majorité de la population).

C'est le cas de la grippe et de 4 coronavirus (cousins du Sars-CoV2). ${ }^{4}$ Grippes, rhinovirus et coronavirus endémiques génèrent chaque année un nombre variable d'hospitalisations et de morts, mais en grande majorité chez les personnes les plus âgées ; la vaccination de la majorité des plus de 65 ans réduit les formes grippales graves ( $\mathrm{SpF}, 2000)$, mais certaines années sont significativement plus sévères tant au niveau mondial (1956-1958, 1968-1970) que national les années 2009, 2014-15, 2016-17-18 ont une mortalité grippale plus forte).

$\mathrm{Du}$ point de vue purement théorique, il est nécessaire d'attendre vingt ans pour estimer moyenne et variance annuelle de la pathogénicité du virus, et juger de son caractère stationnaire et donc endémique.

D'un point de vue pratique, pour le reste des humains, il faut pouvoir caractériser même de manière imparfaite l'état de la transition vers l'endémie, et suivre l'évolution de la pathogénicité à travers divers indicateurs de sévérité empirique.

\section{II) Nouvelle stratégie d'estimation de l'immunité collective et indicateur en temps réel}

Nous cherchons à mesurer l'évolution de l'immunité globale, ou plus précisément de la sévérité empirique du virus, un indicateur qui pourrait guider les politiques publiques.

La sévérité pourrait se mesurer comme le ratio du nombre de nouveaux cas graves (hospitalisation ou décès) sur le nombre de nouveaux cas (nouvelles infections), se celles-ci étaient mesurées de manière fiable et continue.

En l'absence de mesure fiable de l'incidence, la logique immunitaire nous permet d'utiliser directement des données sur les formes sévères (statistiques d'hospitalisation par âge); en l'absence de mesure expérimentale fiable, la stratégie d'estimation la plus classique consiste à utiliser une quasi-expérience que nous décrirons.

\section{Le manque de fiabilité des mesures d'exposition et la nécessité de mesures alternatives}

Estimer en continu le nombre de nouvelles infections aurait été très simple s'il avait été mis en place une collecte auprès d'échantillons représentatifs comme pour les autres phénomènes sociaux ou économiques (Sender, 2021).

Il aurait alors été possible non seulement de calculer de manière fiable l'exposition de la population, mais aussi une mesure directe de la pathogénicité du virus.

\footnotetext{
${ }^{4}$ Faute de tests sanguins ou PCR, grippe, rhume et infections par divers coronas sont souvent confondus en pratique.
} 
Mais les méthodes de collectes utilisées jusqu'à présent ne permettent pas de mesure fiable :

- Les tests PCR sont volontaires, et souffrent d'un biais de participation ; la capacité à tester était insuffisante avant l'automne.

- Les enquêtes sérologiques ne sont pas régulières et ne permettent pas de mise à jour fréquentes des évaluations, sauf à supposer qu'aucune immunité ne se créé et que le ratio de forme sévère reste inchangé dans le temps.

Par ailleurs, ces mesures souffrent de biais importants :

- Petersen and Phillips (2020) estiment à 75\% la proportion d'asymptomatiques lors de la première vague, alors que Santé Publique France fait état d'approximativement 50\% d'asymptomatiques dans les tests PCR.

- Les résultats des études du Diamond Princess, souvent utilisés comme référence (Pasteur, mars 2021) confirment ce biais. Le navire de croisière Diamond Princess, immobilisé pendant deux semaines dans le port de Yokohama, en février 2020, avec ses 3600 passagers et son équipage, a permis, grâce à l'absence totale de mesure d'isolation des passagers, et grâce à un système de ventilation commun des cabines, d'obtenir une exposition de la totalité de ses occupants au virus Sars-CoV2. Pourtant, seuls $20 \%$ des passagers ont été testés positifs.

Comme cela est bien connu dans le cas des vaccins qui estiment à trois semaine le délai pour la montée significative d'anticorps, leur création est progressive après une infection; à contrario, les infections courtes, souvent asymptomatiques, ne permettent pas la création d'anticorps spécifiques détectables par sérologies.

Ioannidis (2020) étudie de nombreux prélèvements sérologiques, et montre la variabilité extrême des estimations des taux de létalité du virus (IFR pour Infection Fatality Ratio) ce qui suggère un manque de robustesse des estimations d'exposition basées sur les mesures sérologiques ; Driscoll et al. (2020) étudient l'exposition par tranche d'âge, et dans certaines régions les estimations d'exposition de la population sont supérieures à $100 \%$.

Préambule : évidence d'une structure d'âge des formes sévères

\section{Taux hospitalisation CoViD \\ \% population tranche d'âge}

- 20-29 ans - 30-59 - $60+$

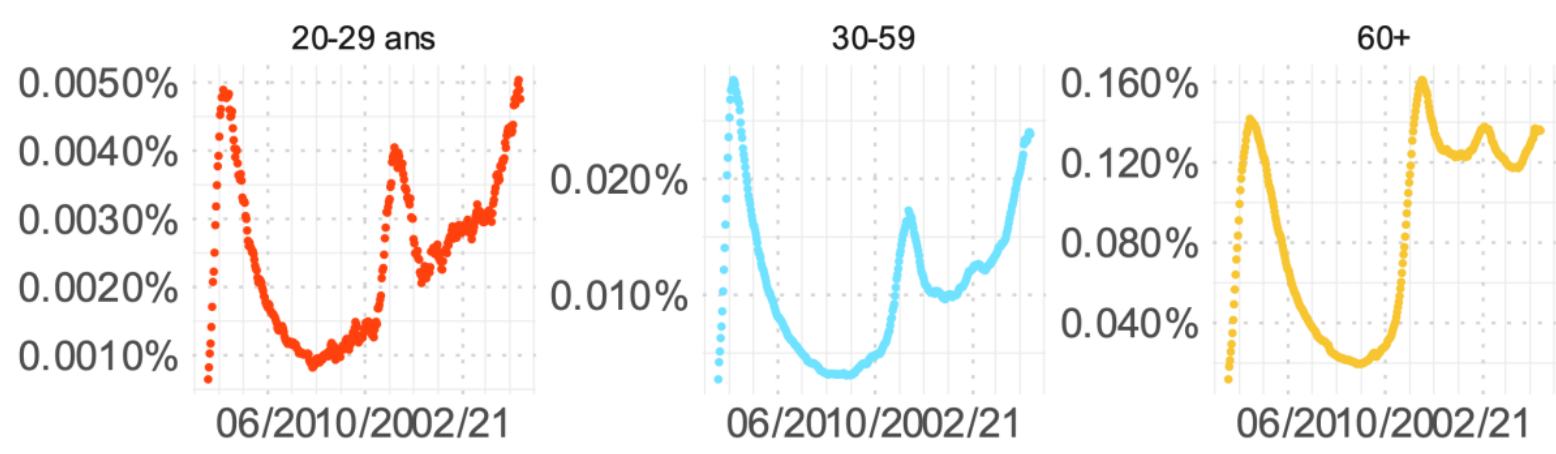


Les données d'hospitalisation montrent que :

- les moins de trente ans possédaient une immunité profonde (les formes graves sont exceptionnelles). L'interprétation naturelle est que l'infection par les coronavirus endémiques qui survient chez $100 \%$ de la population dans l'enfance autour de l'âge de 3 ans protège très bien contre Sars-CoV2

- les plus de soixante ans ont les plus faibles défenses immunitaires : à la baisse naturelle de l'immunité acquise avec le temps et la moindre réactivité du système immunitaire le rendent plus sensible aux variations virales

- la population des 30-59 est dans une situation intermédiaire du point de vue statistique (fragilité «modérée »); ce groupe pourra construire des défenses immunitaires par exposition.

\section{Estimation pour la France}

En l'absence de mesure expérimentale fiable de l'incidence, la stratégie d'estimation la plus classique consiste à utiliser une quasi-expérience. Il s'agit d'identifier une population témoin à la mémoire immunitaire faible, et une population cible à la réaction et mémoire immunitaire forte. Leur comparaison permet de mesurer l'évolution de l'immunité profonde dans la population cible, c'est-à-dire, la réduction de la sévérité empirique compte-tenu du passage du temps et des mutations du Sars-CoV2 ?

Nous prenons comme groupe de référence les plus de 60 ans comme proxy de la population fragile, et les 30-59 ans comme population susceptible de construire des défenses immunitaires. Nous excluons les moins de trente ans dont on peut considérer qu'ils sont largement protégés (mais les prendre en compte aurait peu d'influence sur la mesure).

Notre mesure de la proportion de personnes immunisées, ou de l'évolution de la sévérité empirique du Sars-CoV2, est le simple ratio d'hospitalisation des 30-59 ans sur les hospitalisations des plus de 60 ans..$^{5}$

Les données proviennent de Santé publique France, qui bénéficie du signalement automatique et systématique des cas de Covid dans les hôpitaux (SI-VIC, système d'identification des victimes), mis à disposition quotidiennement sur https://data.gouv.fr

A partir de février 2021, la vaccination progressive des personnes les plus âgées fragilise notre indicateur ; s'il est possible de corriger de l'effet vaccination, la diminution des effectifs des personnes fragiles rend l'indicateur moins robuste, et plus sensible aux hypothèses de correction. Pour supprimer cet effet, il faut prendre en compte non pas les hospitalisations, mais les hospitalisations par tranche d'âge (rapportée à la population) diminuée de l'effet effectif de la vaccination.

La vaccination ne s'est pas faite de manière aléatoire, mais s'est concentrée vers les plus fragiles ; il faut prendre en compte l'effet préventif du vaccin et celui du ciblage.

\footnotetext{
${ }^{5}$ Les graphiques d'hospitalisation par page sont présentés en annexe. En France, lors de la deuxième vague, les hospitalisations des séniors étaient au même niveau qu'au pic de la première vague, alors qu'elles n'étaient que de moitié pour la population active.
} 
Début avril, au moins une dose de vaccin a été injectées à :

- un tiers des plus de 60 ans

- les deux tiers des plus de 75 ans

- $\quad 95 \%$ des résidents en EHPAD et EMS.

Pour quantifier l'effet ciblage, nous faisons l'hypothèse que les personnes vaccinées sont deux fois plus à risque que les personnes non-vaccinées, car pour rappel les probabilités d'évènement COVID grave doublent tous les dix ans.

Pour quantifier l'effet vaccin, nous retenons l'hypothèse de Pfizer (2021) estime que la première injection assure 52\% d'immunité contre les formes graves, et la deuxième $92 \%$.

Nous faisons l'hypothèse d'une semaine de délai.

Le calcul se fait en deux étapes :

- Soit $P_{j}^{V}$ la population effectivement vaccinée au jour j. On a : $P_{j}^{V}=0.92 \cdot V_{j-7}^{2}+$ $0.52 \cdot\left(V_{j-7}^{1}-V_{j-7}^{2}\right)$ où $V_{j-7}^{1}\left(\right.$ resp. $\left.V_{j-7}^{2}\right)$ est le nombre cumulé de personnes ayant reçu une première (resp. deuxième) dose de vaccin au moins une semaine avant la date dans la classe d'âge considérée.

- La vaccination réduit la population d'une proportion $2 \cdot \frac{P_{j}^{V}}{P+P_{j}^{V}}$ où $\mathrm{P}$ est la population de chaque grande tranche d'âge.

France: indicateur de sévérité

(ratio d'hospitalisations

$30-59$ vs $65+)$, corrigé des vaccinations

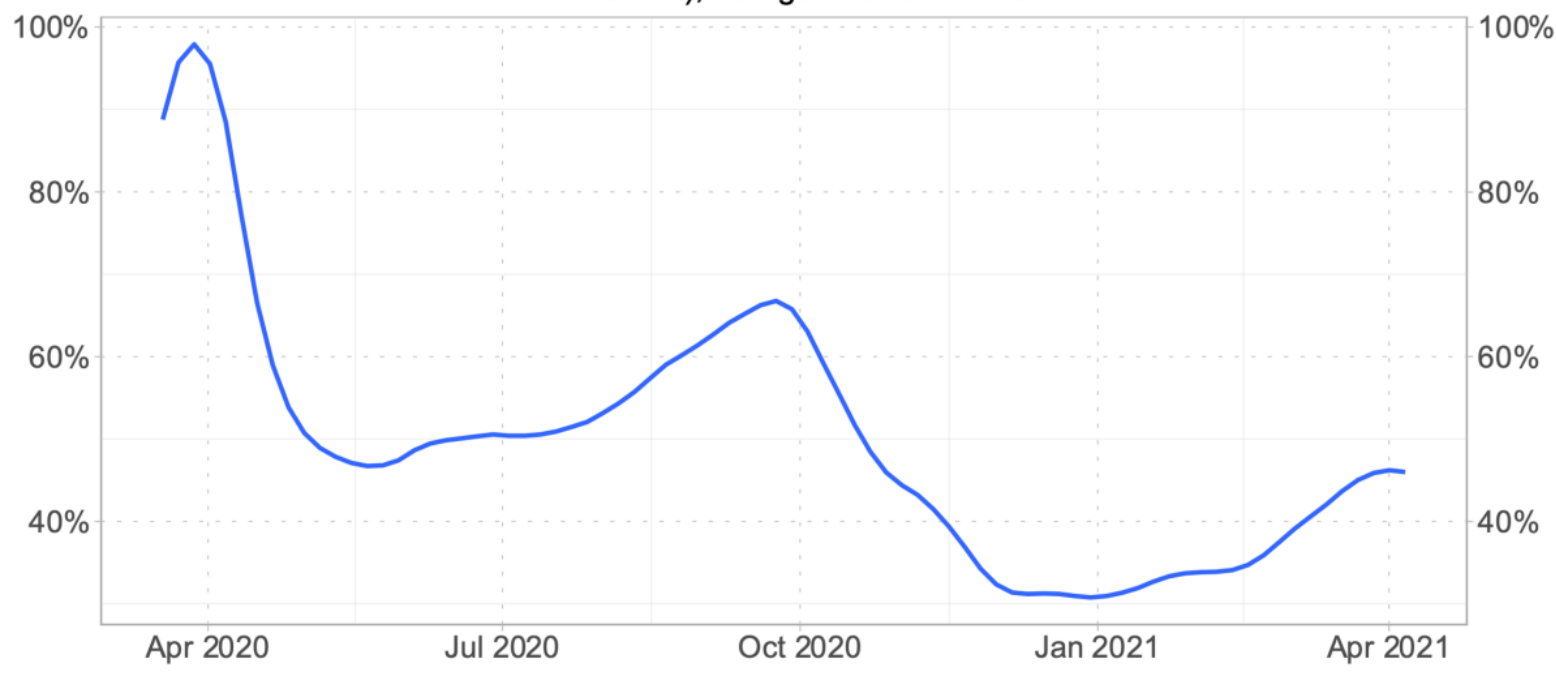

Source des données : Santé Publique France, via data.gouv.fr. Calculs des auteurs. Les graphiques sont mis à jour ici : https://samjs.sjinyapps.io/CoViD

\section{Cohérence et biais de l'estimateur}

Il convient d'analyser la robustesse de cet estimateur, comme nous l'avons fait pour les autres estimateurs utilisés par les organismes officiels.

Notre indicateur n'implique pas la résolution d'un problème «mal posé »; les biais et hypothèses implicites au «problème » qu'il résout en font une borne basse de l'estimation de la progression de l'immunité collective. 
D'une part, les données d'hospitalisations sont plus fiables que celles des PCR, et, contrairement à celles des tests sérologiques, mises à jour quotidiennement. Des problèmes de classification existent cependant, et la diminution de la sévérité devrait logiquement conduire à catégoriser comme patients $\mathrm{CoViD}$ une part toujours plus importante de patients admis en réalité pour d'autres pathologies chez les 30-59 ans qui ont construit des défenses immunitaires.

Par ailleurs, considérer que les seniors n'acquièrent pas de défenses immunitaires, et n'ont pas adopté un comportement plus précautionneux conduit à une sous-estimation de leur protection. ${ }^{6}$

Ces deux facteurs conduisent à une sous-estimation de la progression de l'immunité collective dans la population adulte, et notre estimation des deux tiers est une borne basse.

Cette estimation peut être formalisée comme problème d'estimation, voir Appendice A.2.

\section{Robustesse}

Prendre les 75+ comme catégorie fragile de référence conduit à une estimation de l'immunité collective plus forte, de manière significative aux US (moins en France)

Cet indicateur de la pathogénicité du virus reflète à la fois la montée de l'immunité collective et l'ensemble des facteurs qui influencent la pathogénicité, tels que saisonnalité et mutations virales.

Une hausse modérée de la sévérité est également visible lors de chaque vague par d'autres indicateurs, notamment par un rebond du ratio réanimation sur hospitalisation ${ }^{7}$ que l'on peut observer dans chaque tranche d'âges, et qui serait à comparer avec les variations saisonnières des admissions en réanimation pour les autres virus respiratoires.

\footnotetext{
${ }^{6}$ Les seniors se sont également mieux protégés par ailleurs après la deuxième vague. Les enquêtes CoViPreV en témoignent (gestes barrières tels que port du masque et distanciation sociale plus généralise chez les seniors que dans le reste de la population). Ceci est également visible aux Etats-Unis sur les ratios d'hospitalisation par âge.

${ }^{7}$ Les deux indicateurs sont corrélés sur le court-terme. Depuis la première vague, le ratio réanimation/hospitalisations a baissé en raison de l'amélioration des protocoles de soins et pas uniquement de l'immunisation de la population, il ne peut donc être pris comme mesure alternative de la progression de l'immunité.
} 


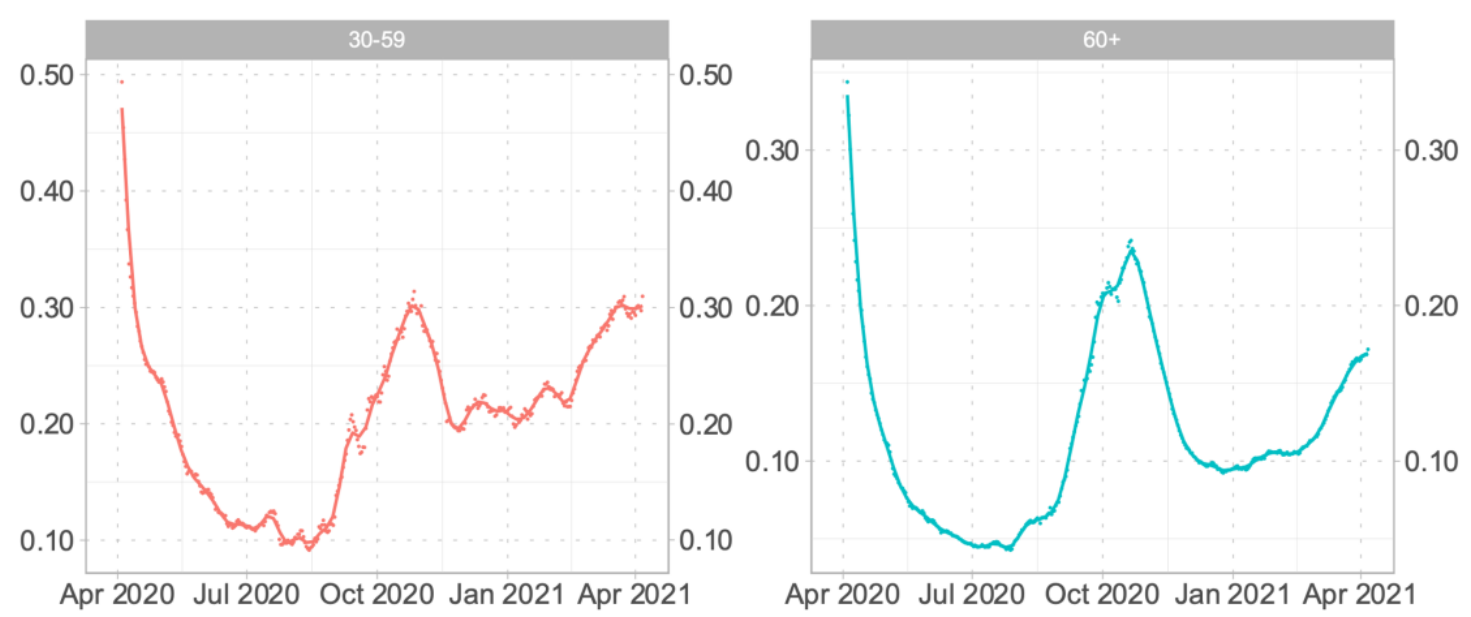

\section{III) Analyse de long-terme : limites et données alternatives}

\section{Limites des données d'hospitalisation}

Si le caractère endémique ne peut se juger qu'avec le passage du temps, une comparaison des caractéristiques et de l'impact du virus avec celles des virus respiratoires endémiques peut fournir des indications précieuses - et à défaut d'une preuve formelle de stationnarité, suggérer l'acceptabilité du virus au regard des normes historiques.

Ainsi :

- Compte tenu de la saisonnalité des infections virales aériennes et de l'échappement immunologique partiel des variants des virus endémiques, on ne peut s'attendre à une baisse de $100 \%$ de notre indice de sévérité.

- On peut en revanche comparer hospitalisations et mortalité aux normes saisonnières historiques.

Bien que cela ne soit pas possible actuellement avec les données d'hospitalisation, cela est partiellement possible avec les données de mortalité.

\section{Séries longues}

Seule la mortalité globale, toutes causes confondues, est disponible en séries longues : ni les hospitalisations globales, ni la mortalité spécifique aux virus respiratoires ne le sont.

Sur cette base, malgré la vague hivernale de contagion, nous sommes déjà revenus dans une situation historiquement normale :

- l'Insee montre que la mortalité globale depuis le premier janvier est inférieure de près de $10 \%$ à celle de la même période en 2019 pour toutes les catégories d'âge jusqu'à 65 ans. 
- les z-scores calculés sur période plus longue par euromomo.eu montrent également que la mortalité est aujourd'hui sur ou sous la tendance historique pour les moins de 65 ans depuis le début de l'année, et pour les plus de 65 ans depuis la mi-mars et l'implémentation significative de la vaccination.

Euromomo, excess mortality (z-score), 45-64 ans, France
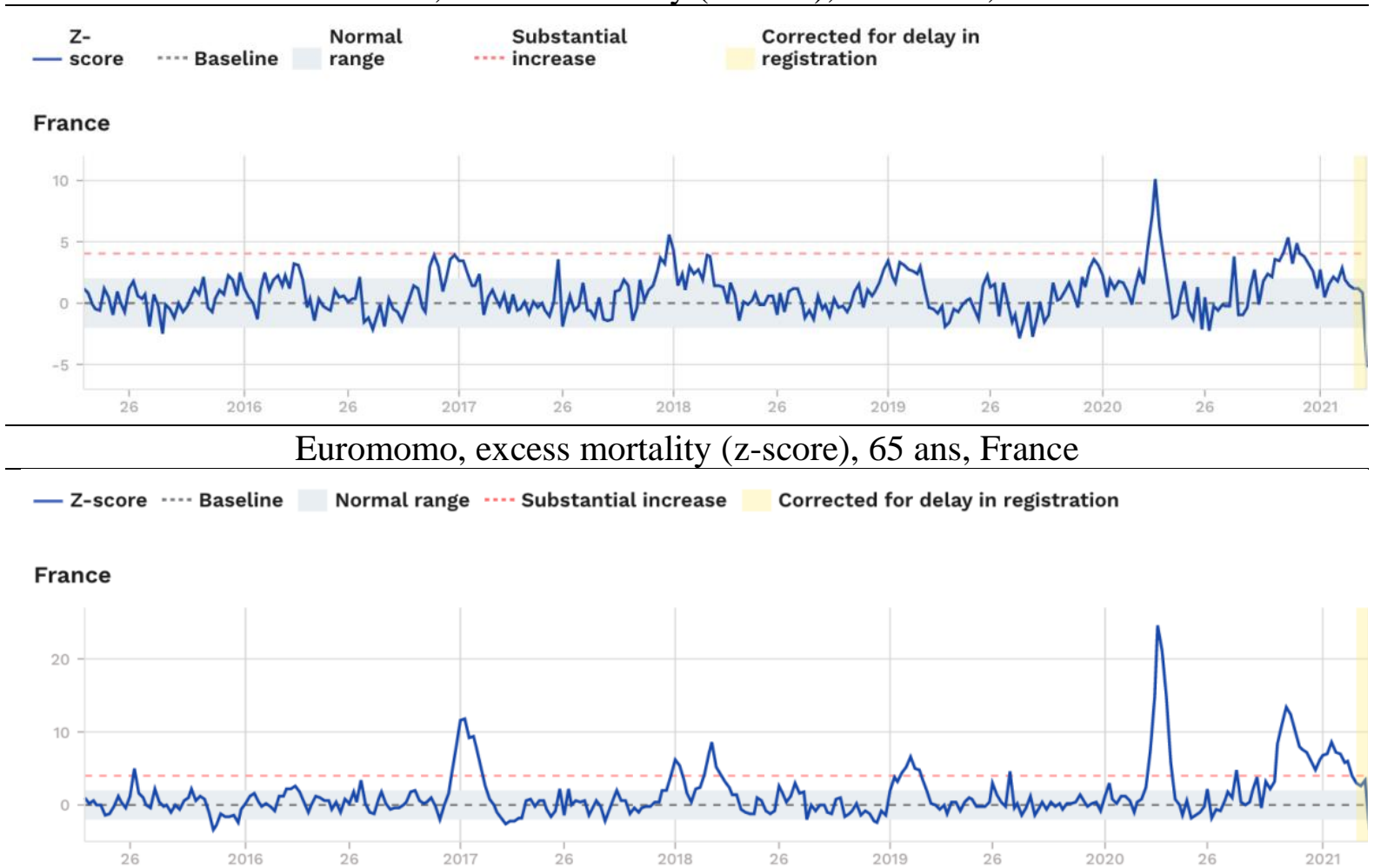

Les années 2017, 2018 et 2019 ont été marquées par une surmortalité hivernale; pour les 45 64 ans, la surmortalité hivernale est moins importante début 2021 qu'en 2017-2019 ; pour les 65 ans et plus, le pic de janvier 2021 est similaire à celui des années 2017-2019.

Source Euromomo: $\quad$ https://www.euromomo.eu/graphs-and-maps\#z-scores-by-country

Méthodologie : https://www.euromomo.eu/how-it-works/reports-and-publications

Même si seul le passage des années permettra d'en juger, ces statistiques suggèrent que le Sars$\mathrm{CoV} 2$ pourrait se fondre dans les coronavirus existant sans engendre de surplus significatif de mortalité (l'alternative étant un état stationnaire avec une mortalité annuelle plus forte).

\section{Conclusion}

Cette étude présente un nouvel indicateur de progression de l'immunité collective dans la population adulte, qui peut être calculé en temps réel dans la majorité des pays.

L'estimation d'une diminution des deux-tiers de la sévérité empirique suggère une progression effective de l'immunité profonde des 30-60 ans du même ordre de grandeur. 
L'estimation de l'exposition des moins de trente ans comme des seniors a moins d'importance pratique, puisque les moins de trente ans étaient en pratique immunisés avant la première vague, et que les seniors restent les plus fragiles et sont appelés à être immunisées par vaccination.

L'affaiblissement récent de la mortalité vers les tendances saisonnières de long-terme, dans toutes les tranches d'âge, tend à confirmer l'hypothèse d'une transition vers une forme endémique, et un Sars-CoV-2 qui pourrait même se fondre dans les virus respiratoires sans en modifier les caractéristiques d'ensemble, ni du point de vue de la mortalité, ni du point de vue de la saisonnalité.

Des hausses saisonnières de sévérité semblent se profiler. Un volume de données et travail plus important serait nécessaire pour analyser les déterminants des vagues épidémiques, avec leurs composants cycliques soit aléatoires soit saisonniers, et pour quantifier de manière plus précise les risques d'échappement à l'immunité naturelle et vaccinale. 


\section{Appendices}

\section{A.2) Saisonnalité des virus aériens et du Sars-CoV2}

La saisonnalité est un phénomène connu de l'ensemble des virus respiratoires. Audi et al. (2020) montrent la saisonnalité des coronavirus endémiques, les conditions atmosphériques, notamment température, humidité et concentration en oxygène jouant tant sur la réplication virale que sur l'efficacité de la réponse immune (voir aussi DoPico et al., 2005).

Dans la période récente, le cycle épidémique de la COVID correspond au cycle classique sous nos latitudes des virus typiques des syndromes grippaux. Les données de syndromic trends montrent ainsi une très forte diminution des détections de l'ensemble des virus associés aux syndromes grippaux, à l'exception notable des rhinovirus.

Une analyse poussée de ces graphiques dépasse le cadre de notre étude. Ils permettent d'illustrer que les poussées de circulation du Sars-CoV2 correspondent au cycle saisonnier classique de circulation grippale, de la mi-octobre à la mi-avril.

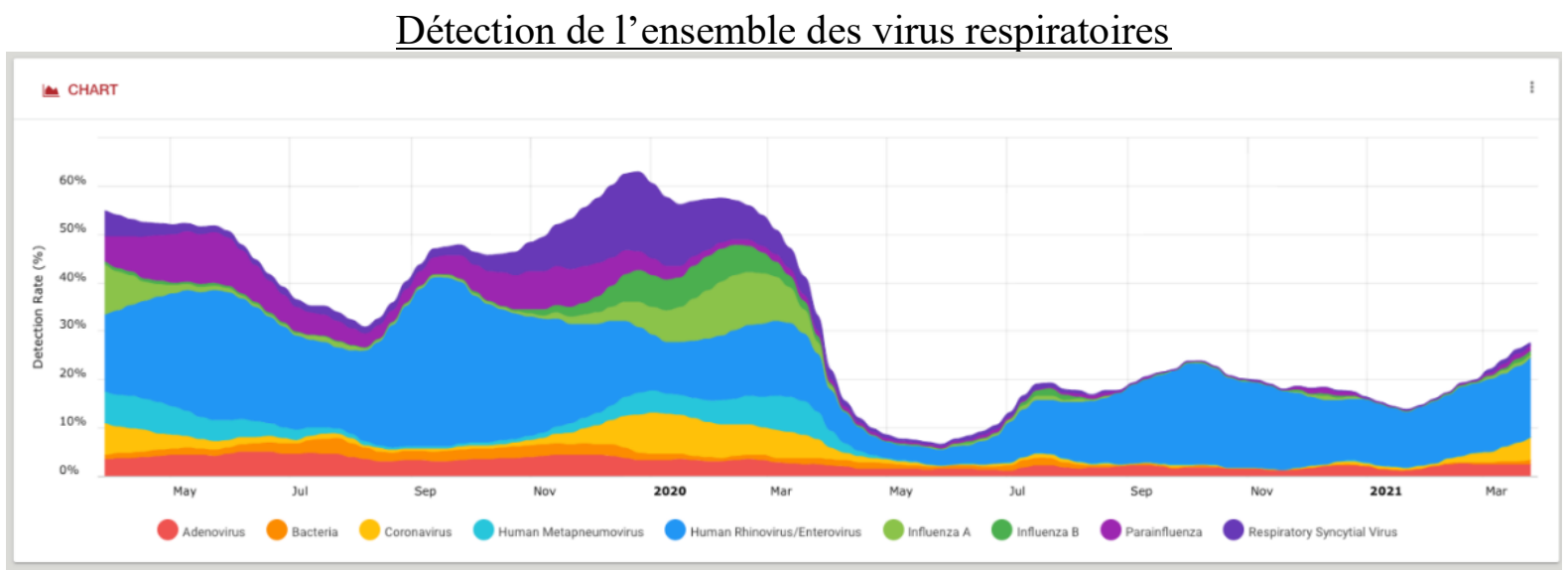

Détection des virus respiratoires, zoom sur des virus en forte diminution

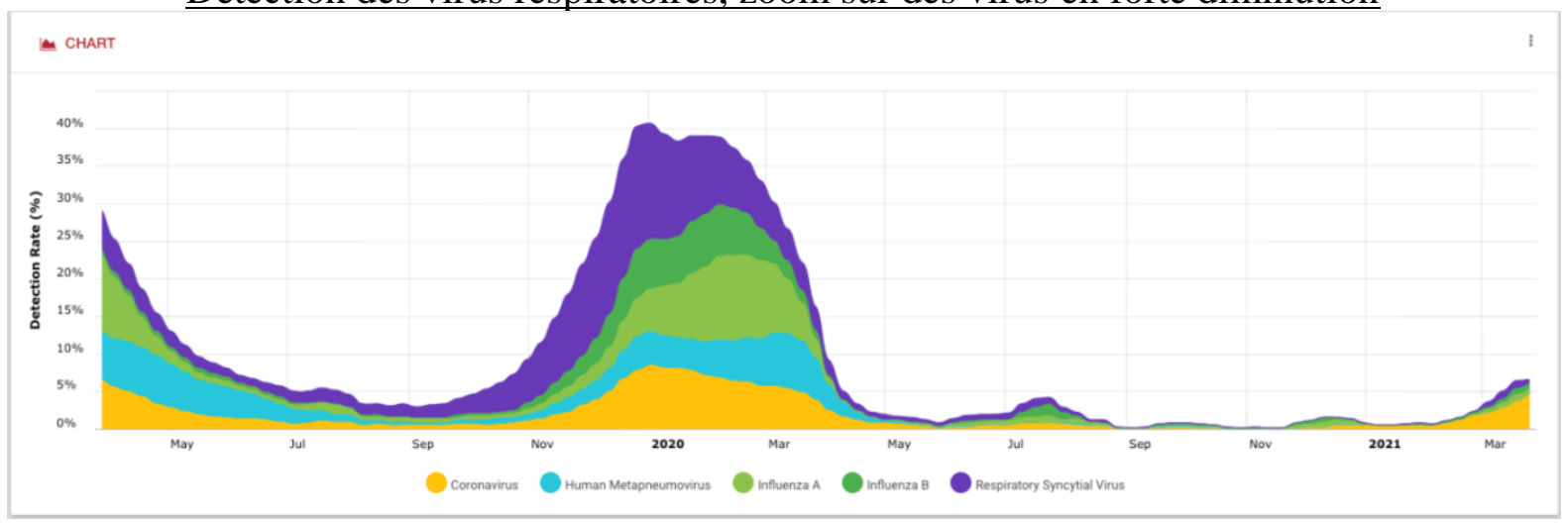

Taux de détection des pathogènes, moyenne centrée sur 3 semaines, Etats-Unis.

Source : Syndromic trends. https://syndromictrends.com Méthodologie : voir Meyers et al. (2018) 


\section{Références}

Audi et al., 2020. Seasonality of Respiratory Viral Infections: Will COVID-19 Follow Suit? Public Health. https://www.frontiersin.org/articles/10.3389/fpubh.2020.567184/full

Carrat et al. 2020. Seroprevalence of SARS-CoV-2 among adults in three regions of France following the lockdown and associated risk factors: a multicohort study. MedRxiv (01). https://www.medrxiv.org/content/10.1101/2020.09.16.20195693v1

Dopico et. al., 2015. Widespread seasonal gene expression reveals annual differences in human immunity and physiology. Nature (6). https://www.nature.com/articles/ncomms8000

Driscoll et al., Nov. 2020. Age-specific mortality and immunity patterns of SARS-CoV2. Nature (590). https://www.nature.com/articles/s41586-020-2918-0

Ioannidis J, 2020. Infection fatality rate of COVID-19 inferred from seroprevalence data. WHO Bulletin (sept 2020). https://www.who.int/bulletin/volumes/99/1/20-265892/en/

Meyers et al., 2018. Automated Real-Time Collection of Pathogen-Specific Diagnostic Data: Syndromic Infectious Disease Epidemiology. JMIR Journal of Public Health and Surveillance (4-3). https://publichealth.jmir.org/2018/3/e59/

Petersen I., Phillips E., Sept. 2020. Three Quarters of People with Sars-CoV-2 Infection are Asymptomatic: Analysis of English Household Survey Data. Clinical Epidemiology (12). https://www.dovepress.com/three-quarters-of-people-with-sars-cov-2-infection-areasymptomatic-an-peer-reviewed-article-CLEP

Pfizer, 2020. Documentation. https://www.vidal.fr/medicaments/gammes/comirnaty100658.html

Santé Publique France, 2015. Impact de la vaccination contre la grippe saisonnière. https://www.santepubliquefrance.fr/maladies-et-traumatismes/maladies-et-infectionsrespiratoires/grippe/documents/article/impact-de-la-vaccination-contre-la-grippe-saisonniere-sur-lamortalite-des-personnes-agees-en-france-de-novembre-2000-a-avril-2009

Segondy, M., 2020. Les Coronavirus humains. Rev Francoph Lab. (526). https://www.ncbi.nlm.nih.gov/pmc/articles/PMC7604068/

Sender, S., 2021. Données de crise ou crise des données ? Linkedin. https://www.linkedin.com/pulse/from-fast-food-data-how-artificial-intelligence-needssender-phd-/

Sonigo, $\quad$ P. 2020. Éléments d'immunologie, Linkedin: https://www.linkedin.com/pulse/comprendre-1\%C3\%A9volution-de-sars-cov2-et-loriginedes-mutants-sonigo/ 